\title{
Attraction et rétention de la main-d'œuvre : Mesure de la problématique et pistes de solutions
}

\author{
François Bernard Malo, \\ Jules Jean-Gilles et \\ Geneviève Vallée \\ (Université Laval)
}

\section{Introduction}

D'ici tout au plus dix ans vous devrez vous pencher de façon sérieuse sur les moyens à mettre en place pour attirer de la main-d'œuvre en vos murs et conserver vos meilleurs employés... ou vous disparaitrez... tout simplement! Avec le vieillissement accéléré de la population active les entreprises québécoises de toutes tailles connaîtront très bientôt de graves problèmes d'attraction et de rétention de leur maind'œuvre hautement qualifiée. Dans un nombre important de PME situées en région ce problème est déjà bien connu. Cet

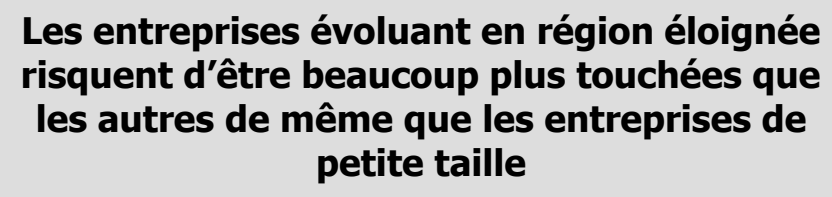

Les entreprises évoluant en région éloignée risquent d'être beaucoup plus touchées que les autres de même que les entreprises de petite taille

article a pour ambition d'expliquer en quoi consiste un problème d'attraction et de rétention de la maind'œuvre et comment il est possible d'y faire face.

\section{Développement}

Dans la littérature en Sciences de la gestion il est plutôt difficile de trouver un sujet sur lequel tous les auteurs sont unanimes. La question du vieillissement accéléré de la population active dans les sociétés dites développées et ses impacts sur les difficultés d'attraction et de rétention de la maind'œuvre hautement qualifiée en est l'une des plus belles exceptions. Depuis quelques années on ne compte plus le nombre d'articles scientifiques et d'ouvrages de vulgarisation qui ont été publiés sur la question. Une simple revue de la littérature nous a permis d'en identifier quelques milliers.

\section{Quand peut-on parler d'un problème d'attraction et de rétention?}

On peut dire qu'une organisation rencontre un problème d'attraction et de rétention de sa maind'œuvre dès lors qu'elle n'est plus capable d'attirer et de conserver à son service les employés dont elle a besoin pour accomplir ses objectifs et réaliser sa mission. Précisons dès maintenant que bien souvent ce qui entraîne le départ volontaire des employés d'une organisation est aussi ce qui limite sa capacité à attirer de nouvelles candidatures.
Trois précisions s'imposent et découlent directement de cette définition. Premièrement il est important de noter que ce ne sont pas toutes les entreprises qui vivront un problème d'attraction et de rétention de la maind'œuvre et que parmi celles qui en vivront un, ce ne sont pas tous les employés de cette organisation qui seront également concernés par la problématique. Les entreprises évoluant en région éloignée risquent d'être beaucoup plus touchées que les autres de même que les entreprises de petite taille incapables d'offrir à leurs employés des conditions de travail au moins équivalentes à celles consenties par les grandes organisations. En ce qui a trait à la maind'œuvre touchée par la problématique les recherches ont bien en évidence que les problèmes sont davantage associés aux travailleurs hautement qualifiés, maîtrisant les compétences au cœur de la stratégie d'affaires de l'organisation et que la situation est d'autant plus problématique que ces travailleurs occupent des emplois difficilement remplaçables par des moyens technologiques ou des procédés d'externalisation de la production ${ }^{1}$. 
Deuxièmement, en fonction de sa stratégie organisationnelle il est permis de penser que les solutions possibles pour corriger la situation ne seront pas les mêmes d'une organisation à l'autre. Si une organisation donnée cherche à se positionner sur son marché par le biais d'une stratégie de prix inférieurs à ses concurrents, elle devra vraisemblablement miser sur des solutions peu coûteuses, voire même un nombre faiblement élevé de solutions destinées essentiellement à certaines catégories bien particulières d'employés car la majorité de ses employés ne posséderont alors que de faibles qualifications. De façon générale il est même permis de penser que ce type d'organisation cherchera délibérément à avoir un taux de roulement de son personnel supérieur à la moyenne ${ }^{2}$. À l'opposé, si une organisation a fait le choix de se positionner stratégiquement sur son marché par le biais d'une stratégie de domination par une plus grande qualité et plus d'innovations dans ses produits et services, les modèles théoriques en gestion stratégique des ressources humaines de même que la théorie économique de la firme prédisent que l'organisation aura alors besoin d'une main-d'œuvre beaucoup plus qualifiée et qu'elle cherchera à en diminuer le taux de roulement afin de ne pas perdre ses investissements immatériels. En ce sens, l'organisation en question n'hésitera pas à mettre sur pied diverses solutions aux coûts élevés mais qui ont véritablement la capacité de s'assurer que le capital intellectuel détenu par les employés demeure au sein de l'organisation et se renouvelle sans problème. Comme les clients sont 'prêts' à payer pour des produits et services se démarquant par des niveaux de qualité et d'innovation supérieurs, l'organisation n'hésitera pas à se doter de moyens plus 'luxueux' voire plus nombreux ${ }^{3}$.

Il est permis de penser que les solutions possibles pour corriger la situation ne seront pas les mêmes d'une organisation à l'autre.

Troisièmement, il importe de remarquer qu'il existe des liens étroits entre la capacité de rétention de la main-d'œuvre d'une organisation et la satisfaction de sa clientèle. Une recherche récente de Zimmerman et al. (2002) conduite dans diverses unités de soins d'un centre pour personnes âgées a démontré que là où le taux de roulement de la main-d'œuvre est supérieur à la moyenne (à cause de divers facteurs venant miner la satisfaction au travail et entraîner chez les employés des départs volontaires en plus grand nombre), les patients hospitalisés y contractent davantage d'infections nosocomiales et $\mathrm{y}$ décèdent beaucoup plus.

\section{Que faire pour lutter contre un taux de roulement excessif de la main-d'ouvre?}

Supposons que vous en êtes venus à la conclusion que le taux de roulement de ' $X$ ' catégorie d'individus au sein de votre organisation est problématique, comment est-il maintenant possible de lutter contre le phénomène? Idéalement toute tentative de lutter contre un taux de roulement excessif de la main-d'œuvre devrait reposer sur une enquête approfondie permettant de comprendre les principaux motifs évoqués par les employés ayant choisi de quitter volontairement l'organisation. Cette façon de faire renvoie à ce que les auteurs appellent 'les entrevues de départ'. Malheureusement, dans un nombre très important d'organisations, de telles entrevues ne sont pas monnaie courante. De plus, les recherches menées sur les entrevues de départ révèlent que bien souvent les motifs fournis par les employés ne sont pas véridiques.

Que faut-il faire alors? Le premier réflexe de tout bon gestionnaire devrait consister à distribuer aux employés 'restants' un questionnaire à l'intérieur duquel il leur est demandé s'ils ont l'intention de quitter l'organisation au cours de la prochaine année et si oui, quels sont les principaux motifs évoqués pour ce faire. Selon les organisations en question le questionnaire pourra être fermé ou ouvert et même inclure des suggestions que les gestionnaires pourraient prendre en compte pour augmenter le degré d'engagement des employés et ainsi réduire le roulement volontaire de la main-d'œuvre. Cette stratégie, même si à certains égards elle peut ouvrir la porte à une sorte de surenchère des conditions de travail, peut très bien arriver à lutter contre un taux de roulement volontaire élevé. Surtout, elle a l'indéniable avantage de faire prendre conscience aux gestionnaires qu'un employé qui a l'intention de quitter une organisation le fait souvent pour plusieurs raisons. 
Si vous choisissez de ne pas distribuer à vos employés restants un tel questionnaire (question de ne pas ouvrir de boîte de Pandore par exemple), il vous reste une autre solution... fort heureusement! Elle consiste à procéder à un auto-examen de vos différentes pratiques de gestion pour voir si certaines d'entre elles ne poseraient pas problème. Pour réaliser cet exercice, bien entendu, vous devriez vous adjoindre les services d'un consultant spécialisé dans la question qui constituera à son tour un groupe de travail ad hoc composé de gestionnaires de votre organisation et peut-être aussi de quelques représentants de vos employés. Dans l'un ou l'autre de ces cas, plusieurs des éléments qui ressortiront se retrouveront fort probablement dans le tableau suivant traduit de l'ouvrage de Ahr et Ahr (2000) et qui expose les 40 raisons les plus souvent mentionnées par les employés lors des entrevues de départ. Les douze premières réponses figurant dans le tableau 1 seraient particulièrement fréquentes selon Giacalone et Duhon (1991) cités dans Ahr et Ahr (2000).

Tableau 1 : Liste des motifs les plus souvent invoqués par les employés ayant décidé de quitter volontairement leur organisation

\begin{tabular}{|c|c|}
\hline 1. Manque d'opportunités d'avancement & 21. Défis liés à l'emploi \\
\hline 2. Localisation géographique de l'emploi & 22. Élimination de l'emploi occupé \\
\hline 3. Relations avec le supérieur immédiat & 23. Responsabilités liées au travail \\
\hline 4. Contenu du travail & 24. Sécurité d'emploi \\
\hline 5. Stress lié au travail & 25. Manque d'autonomie professionnelle \\
\hline $\begin{array}{l}\text { 6. Règles \politiques\procédures en vigueur au } \\
\text { sein de l'organisation }\end{array}$ & $\begin{array}{l}\text { 26. Manque de ressources pour accomplir son } \\
\text { travail de manière satisfaisante }\end{array}$ \\
\hline $\begin{array}{l}\text { 7. Évaluation du rendement et méthode } \\
\text { d'évaluation du rendement }\end{array}$ & $\begin{array}{l}\text { 27. Manque d'opportunités de développement } \\
\text { professionnel }\end{array}$ \\
\hline $\begin{array}{l}\text { 8. Relations interpersonnelles avec les collègues } \\
\text { de travail }\end{array}$ & 28. Faible sentiment de réalisation personnelle \\
\hline 9. Salaire et rémunération globale & $\begin{array}{l}\text { 29. Absence de reconnaissance pour le travail } \\
\text { accompli }\end{array}$ \\
\hline 10. Formation reçue & 30. Culture organisationnelle \\
\hline 11. Qualité des gestionnaires supérieurs & 31. Relocalisation de l'organisation \\
\hline 12. Conditions de travail en général & $\begin{array}{l}\text { 32. Changement dans les plans de carrière person- } \\
\text { nels }\end{array}$ \\
\hline 13. Avantages sociaux & 33. Raisons médicales ou physiques \\
\hline $\begin{array}{l}\text { 14. Temps et distances nécessaires pour se rendre } \\
\text { au travail }\end{array}$ & 34. Problèmes divers associés au travail \\
\hline 15. Inquiétude quant au futur de l'organisation & $\begin{array}{l}\text { 35. Relocalisation géographique entraînée par le } \\
\text { travail du conjoint }\end{array}$ \\
\hline 16. Peur de voir son poste être éliminé & 36. Façon d'être traitée au sein de l'organisation \\
\hline 17. Équipement inadéquatlobsolètelinsuffisant & 37. Contenu du travail \\
\hline $\begin{array}{l}\text { 18. Cheminement de carrière non approprié ou } \\
\text { absent }\end{array}$ & 38. Environnement de travail \\
\hline $\begin{array}{l}\text { 19. Rétroaction inexacte ou insuffisante au sujet du } \\
\text { travail accompli }\end{array}$ & 39. Équilibre vie professionnellelvie personnelle \\
\hline $\begin{array}{l}\text { 20. Changement involontaire des responsabilités } \\
\text { professionnelles }\end{array}$ & 40. Autres raisons personnelles \\
\hline
\end{tabular}

Abordant la question sous l'angle de l'envie de rester au sein de l'organisation, Capelli $(2002,75)$ (qui précise d'ailleurs que les raisons invoquées par les employés pour rester au sein de leur organisation peuvent grandement varier d'un pays à l'autre) insiste sur cinq facteurs lui apparaissant particulièrement cruciaux :
1- fierté de travailler pour l'organisation en question (car ses gestionnaires sont jugés compétents, possèdent une vision claire du futur de l'entreprise et sont capables de l'atteindre grâce à des stratégies appropriées qui ont le don de motiver les employés); 
2- présence d'un superviseur respecté par ses employés et qui leur apporte du support lorsqu'ils en éprouvent le besoin;

3- rémunération (directe et indirecte) équitable de même que possibilités nombreuses d'apprendre, de croître et de se développer;

4- opportunité de travailler avec des collègues respectés et compatibles avec soi;

5- travail satisfaisant et motivant qui a un sens et qui correspond aux intérêts réels des employés.

Lorsque l'employé prend conscience que ces cinq éléments ne sont pas (ou ne sont plus) présents au sein de l'organisation, il aurait alors tendance à vouloir la quitter. Loin de s'en tenir à ces quelques idées, l'auteur stipule que dans la vaste majorité des cas les employés quittent une organisation pour les mauvaises raisons et cela, pour découvrir plus ou moins tardivement que dans la nouvelle organisation pour laquelle ils oeuvrent les mêmes situations qui les ont amenés à quitter leur précédent employeur s'y retrouvent elles aussi. Il revient donc, à son avis, aux supérieurs immédiats informés du désir de leurs employés de quitter l'organisation de bien les informer des possibilités de remédier à leurs insatisfactions. Selon lui, la clé d'une bonne rétention de la main-d'œuvre réside dans le fait d'avoir un supérieur immédiat sur lequel on peut compter et en qui on peut avoir confiance.

À certains égards on pourrait croire que les raisons invoquées pour les employés pour quitter leur organisation portent en elles-mêmes les pistes de solution pour diminuer le roulement de notre maind'œuvre. C'est vrai mais seulement en partie. En pratique, il existe des tas d'autres façons de s'assurer que nos employés restent chez nous! $\mathrm{Ce}$ sont ces diverses solutions qui seront présentées succinctement dans les lignes qui suivent.

\section{Les diverses façons de lutter contre le rou- lement volontaire de la main-d'œuvre}

Selon les divers résultats des recherches empiriques publiées à ce jour, de même que les conseils émis dans leurs ouvrages par divers consultants spécialistes de la question de l'attraction et de la rétention de la main-d'œuvre, il existe une pléthore de solutions pour s'assurer que les employés trouvent, au sein de leur organisation, les motifs suffisants pour y de- meurer et parviennent à convaincre certaines de leurs connaissances à joindre leurs rangs. De même, il existe aussi plusieurs manières de présenter ces diverses solutions. Parmi ces façons de présenter les diverses solutions disponibles, nous pouvons souligner le regroupement par thèmes 'à la mode' (comme la conciliation travail-famille ou l'éthique), le regroupement en fonction des caractéristiques propres à chaque groupe d'individus (voir par exemple Fields (2001) et Sturges (1998) cités dans Woodruffe 1999), le regroupement en fonction des étapes de carrières atteintes par les divers individus que l'on veut attirer ou retenir (voir par exemple Dychtwald, Erickson et Morison 2006), le regroupement en fonction des coûts des solutions envisagées, le regroupement en fonction des diverses sciences à la base des relations industrielles (voir par exemple Osterman 1987) et enfin, le regroupement en fonction des diverses pratiques de GRH. C'est ce dernier regroupement, constitué au total de douze familles, que nous avons choisi pour la suite de notre travail.

La première famille de solutions possibles pour attirer et retenir davantage la main-d'œuvre a trait aux pratiques de recrutement de l'organisation. Les divers auteurs consultés soulignent l'importance de bien cibler les personnes que l'on veut rejoindre, de leur communiquer clairement nos attentes à leur endroit et cela, dès le départ et enfin, de s'assurer qu'elles soient non seulement capables de faire le travail demandé mais qu'elles soient compatibles avec la culture de l'organisation et ses principales valeurs. Se faisant, les organisations auraient tout avantage à courtiser leurs anciens employés ayant quitté volontairement l'organisation. Malheureusement, pour les personnes déjà à l'emploi de l'organisation, cet éventail de solutions arrive assurément trop tard.

\section{La clé d'une bonne rétention de la main- d'œuvre réside dans le fait d'avoir un supé- rieur immédiat sur lequel on peut compter et en qui on peut avoir confiance.}

La deuxième famille de solutions possibles, n'étant pas non plus très aidante pour les employés actuels, est celle qui regroupe les diverses activités de sélection du personnel. Les auteurs recensés insistent 
cette fois sur l'importance de bien préparer et bien conduire l'entrevue de sélection. Faire preuve de négligence à cet endroit revient à ne pas voir que le candidat à un poste donné peut ne pas convenir. Tout en nous invitant à faire preuve d'ouverture à l'endroit de candidats différents, les auteurs nous rappellent que dans certains cas il peut être intéressant de ne pas chercher à recruter le meilleur d'entre tous. Les meilleurs employés étant naturellement beaucoup plus sollicités que ceux offrant un rendement inférieur. Enfin, précisent-ils, il ne faut pas oublier de vérifier dans quelle mesure le candidat éprouve une réelle satisfaction à accomplir le type de tâches qu'il aura à assumer dans le cadre de son emploi. Afin de prévenir tout roulement excessif de la main-d'œuvre il convient donc de bien sélectionner nos futurs employés en n'étant ni trop exigeants ni trop permissifs.

La troisième famille de solutions possibles pour augmenter son pouvoir d'attraction et sa capacité de rétention regroupe les pratiques liées à l'accueil et à l'intégration des nouveaux venus. Selon les divers auteurs consultés, il convient tout d'abord de mettre sur pied un processus d'accueil et d'intégration bien structuré et qui permettra à la recrue d'être rapidement mise en contact avec des collègues de qualité, compatibles avec elle et pas trop différents en termes démographiques. Par le biais d'activités diverses, le nouveau venu sera incité à tisser de nouvelles relations et créer de nouvelles attaches avec les membres de l'organisation. Cela sera d'ailleurs d'autant plus facile que le lieu du travail sera près de l'endroit où il souhaite habiter. Malheureusement, encore une fois, la plupart de ces idées ne sont pas applicables aux employés déjà à notre service.

La quatrième famille de solutions possibles est sans doute celle qui regroupe le plus d'idées de même que les idées les plus novatrices. Il s'agit de la famille des pratiques de rémunération et de reconnaissance du travail accompli. Selon Block, Claffey, Korow et McCaffrey (2005) la rémunération globale est le deuxième facteur en importance pour expliquer l'insatisfaction au travail. Selon plusieurs autres auteurs s'étant penchés sur le cas spécifique des infirmières (Lum et al., 1998; Chan et Morrison, 2000; Kunaviktikul, Nuntasupawat, Srisuphan et Booth, 2000), la satisfaction à l'égard du salaire reçu influence directement la satisfaction au travail qui agit à son tour sur l'engagement organisationnel et puis sur l'intention de quitter l'organisation. Il est donc extrêmement important de s'en préoccuper d'autant plus que pour cet ensemble de pratiques il n'est pas trop tard pour bien faire les choses! Commençons donc par les idées 'classiques' : offrir une rémunération avantageuse par rapport à la concurrence, soucieuse d'équité interne et externe et en lien avec la performance de chacun, ponctuée d'augmentations régulières, bonifiée par des possibilités de participer aux profits de l'entreprise, agrémentée d'avantages sociaux classiques (congés annuels payés, caisse de retraite, assurance santé, assurance médicament, soins dentaires, massage, chiropractie, acupuncture etc.). En ce qui a trait à la reconnaissance, les gestionnaires devraient s'assurer de remercier les employés pour les bons coups réalisés.

Pour ce qui est des idées 'novatrices', les auteurs suggèrent quelques pistes intéressantes : mettre sur pied un système de rémunération dédié uniquement aux travailleurs susceptibles de prendre leur retraite ou qui possèdent un savoir unique; mettre sur pied un système de rémunération qui ne favorise pas le maintien artificiel en emploi des employés capables et désireux de réorienter leurs carrières; bannir les systèmes de rémunération reconnaissant les performances individuelles pour les remplacer par des systèmes liés aux performances des équipes de travail; tempérer les écarts de salaires entre les gestionnaires supérieurs et les employés de la base; remboursement des frais de pension, d'entretien et de vétérinaires pour les animaux de compagnie, remboursement de frais d'études non liés à l'emploi actuel ou à venir; remboursement des frais d'abonnement, de déplacement et de participation à des organisations dévouées à la promotion de la santé et à l'entretien de saines habitudes de vie (Minçavi; Alcooliques Anonymes; Narcotiques Anonymes; maisons de désintoxication etc.); remboursement des frais d'abonnement et de participation à des associations sportives, culturelles et sociales (club de marche et de plein air; association d'astronomes amateurs; club d'échecs; club pour personnes seules etc.); remboursement de frais d'abonnement et de participation à des groupes de méditation et de relaxation; remboursement des frais liés à l'adoption internationale d'enfants; remboursement des frais liés à l'entretien de la maison, remboursement des frais liés à l'entretien et à la 
réparation des véhicules personnels; remboursements des frais liés à la garde des enfants et à leur éducation; prêt hypothécaire garanti par l'entreprise à un taux plus avantageux que celui normalement offert par les institutions bancaires; distribution de billets gratuits pour assister à des spectacles sportifs ou culturels; système de rémunération 'à la carte' où les employés peuvent choisir un salaire annuel moins élevé de même que certains avantages sociaux garantis dès la première année ou un système de rémunération avec un salaire annuel régulier sans avantages sociaux mais qui comporte un bonus pouvant équivaloir à $120 \%$ du salaire annuel à la fin de la $3^{\mathrm{e}}$ année de service.

En ce qui a trait à la reconnaissance, les auteurs suggèrent de faire très attention aux mots utilisés dans l'organisation afin de ne pas laisser sousentendre à certains employés qu'ils sont moins importants que les autres. Par exemple, il conviendrait de bannir les expressions 'employé en formation', 'employé surnuméraire', 'employé temporaire', 'employé en période probatoire' etc. De plus, il faudrait s'assurer que nos meilleurs employés aient régulièrement contact avec les dirigeants supérieurs de l'organisation et ses principaux clients. Ils pourraient à ce titre faire d'excellents ambassadeurs et servir de modèles à plusieurs. Enfin, certains auteurs suggèrent de solliciter la participation des membres de la famille des employés pour agir à titre d'acteurs ou de mannequins dans les publicités de l'organisation. Les solutions possibles liées aux pratiques de rémunération et de reconnaissance des acquis ont pour principal inconvénient d'entraîner des déboursés importants pour l'organisation et de toucher à la délicate question de l'équité. En ce sens, modifier ces diverses pratiques est toujours possible mais c'est loin d'être un jeu d'enfant.

La pratique de GRH qui est l'évaluation du rendement constitue la cinquième famille de solutions possibles à une problématique d'attraction et de rétention de la main-d'œuvre. Dans cette famille les auteurs insistent pour rappeler que l'évaluation du rendement devrait toujours être menée de manière sérieuse, notamment à la fin de la période probatoire. De plus, il faudrait toujours l'utiliser comme une occasion d'identifier, avec l'employé en question, les bons coups réalisés et les éléments à retravailler, notamment grâce à la formation. Grâce aux évaluations à 360 degrés il pourrait même être don- né aux employés l'occasion de s'exprimer sur les performances de leur supérieur immédiat. En leur communiquant ainsi leur appréciation ils pourraient eux aussi être amenés à se développer et à mieux répondre aux attentes de leur personnel. Pour les nouveaux employés certains auteurs suggèrent notamment de leur communiquer des objectifs faciles à réaliser. De cette façon, ils auront plus facilement tendance à développer un attachement affectif à l'égard de l'organisation et ils seront moins enclins à vouloir la quitter.

La sixième famille de solutions possibles peut être regroupée sous la pratique 'formation'. Dans cette famille il est bien entendu question de s'assurer que les employés de l'organisation soient parfaitement compétents pour faire le travail qui leur est demandé et celui qui viendra. C'est en ce sens que certains auteurs parlent de l'importance de développer les compétences larges des employés, de telle sorte qu'ils soient capables de s'adapter au changement rapidement et avec facilité. Bien entendu, ceci implique que les besoins de formation ont été très bien identifiés et répondent à un besoin réel aussi bien de la part de l'organisation que des employés. Parmi les formations à dispenser, les entreprises auraient avantage à former leurs gestionnaires pour qu'ils apprennent de plus en plus à devenir des coachs $\backslash$ facilitateurs et qu'ils contribuent à faire en sorte que toute l'entreprise devienne elle-même intelligente et apprenante. Du même coup, il conviendrait aussi de les sensibiliser à l'importance de contrôler le taux de roulement de leurs employés et à la nécessité de faire preuve de prudence avant de procéder à de nouvelles embauches dont l'organisation pourrait devoir se départir à court terme. En complément, comme le goût d'apprendre se cultive, quelques auteurs suggèrent aussi aux entreprises de partager leurs connaissances avec leurs employés (notamment dans la planification budgétaire à des fins personnelles) et d'inciter ses derniers à suivre divers cours qui les intéresse... peu importe qu'ils soient ou non en lien direct avec leur emploi. Les liens pourront éventuellement se faire, même de façon inconsciente et indirecte.

La septième famille de solutions possibles a trait à l'organisation du travail et est un peu comme la famille des pratiques de rémunération et de reconnaissance du travail accompli. Elle comporte plusieurs idées dont certaines peuvent être qualifiées de 
'classiques' et d'autres de 'novatrices'. Parmi les idées 'classiques' plusieurs auteurs parlent d'enrichissement et d'élargissement du travail, de rotation volontaire des postes de travail, d'aménagement flexible des horaires, de travail en équipe et de charge de travail 'raisonnable'. Parmi les idées novatrices on compte notamment sur des politiques d'arrangement des temps de travail permettant aux personnes retraitées de revenir au boulot à leur rythme et au moment qui leur convient, de constituer des groupes de travail dont la taille correspond aux besoins et aux attentes des employés et de journées officielles de rotation des tâches lchangement de responsabilités où certains employés sont invités à venir passer une journée entière dans les chaussures de quelqu'un d'autre et à le suivre pas à pas. Visant aussi bien à briser l'ennui qu'à tisser de nouvelles relations entre les individus, ces diverses solutions contribuent toutes, à leur manière, à solidifier le collectif de travail et de là, à atténuer les problèmes d'attraction et de rétention de la main-d'œuvre.

\section{Une organisation où règne une surcharge de travail importante et continue est souvent une organisation où les problèmes d'attraction et de rétention de la main- d'œuvre sont des plus importants.}

La huitième famille de solutions cadre dans ce que l'on appelle les pratiques de conciliation vie professionnelle/vie personnelle si chères aux représentants des générations X et Y (Holbeche 1998 et Sturges 1998 cités dans Woodruffe 1999). Dans cette famille on retrouve les pratiques d'aménagement des temps de travail réservées aux personnes ayant des obligations familiales particulières à assumer (jeune enfant, parent malade etc.), les services de transport du domicile au travail assurés par des bus de l'entreprise, les services de nettoyage des vêtements personnels, les services d'entretien et de nettoyage de la maison et de la voiture personnelle, les services d'épicerie, l'accompagnement dans la préparation de documents légaux (comme les rapports d'impôt, la rédaction testamentaire, l'achat d'une propriété), l'assistance professionnelle pour des raisons personnelles (comme l'aide psychologique pour la famille, l'aide à l'adoption internationale, l'aide pour les couples en difficulté, les conseils d'un orienteur...), l'assistance médicale sur les lieux du travail (infirmière, médecin, dentiste, chiropraticien), l'assistance 'beauté' sur les lieux du travail (coiffure, maquillage, pédicure...), l'aménagement de locaux pour recevoir sur les lieux du travail les enfants, les parents à charge et les animaux de compagnie et enfin, l'aménagement d'espaces sur les lieux du travail pour allaiter les enfants, faire la sieste et se distraire (notamment par la constitution d'une salle de massage, d'une librairie, d'un parc et d'un café internet). En bref, ce que mettent en évidence ces diverses idées c'est que l'entreprise peut aider ses employés à assumer leur rôle de parent et de citoyen en leur offrant toutes sortes d'occasions d'être appuyés et distraits par l'organisation qui les emploie.

La neuvième famille de solutions possibles pour juguler un problème d'attraction et de rétention de la main-d'œuvre a trait aux pratiques de santé et sécurité au travail. Aux dires des divers auteurs consultés il est plus qu'important que les gestionnaires s'assurent que l'organisation soit un lieu sain et sécuritaire aussi bien pour la santé physique que psychologique des employés. Une organisation où règne une surcharge de travail importante et continue est souvent une organisation où les problèmes d'attraction et de rétention de la main-d'œuvre sont des plus importants. Pour prévenir ces problèmes les gestionnaires auraient tout avantage à créer un comité paritaire de prévention des accidents du travail qui aurait notamment pour tâche d'évaluer, de manière régulière, la présence des risques à la santé et d'y trouver des remèdes.

La dixième famille de solutions a trait à ce que l'on appelle communément la gestion des carrières. Comme la question de la conciliation vie professionnelle/vie personnelle, la gestion des carrières semble être une préoccupation extrêmement importante pour les représentants des générations $\mathrm{X}$ et $\mathrm{Y}$. Dans cette famille de solutions on retrouve les politiques de promotion favorisant l'embauche à l'interne, la diffusion régulière de toutes les possibilités de promotion et de mutation auprès des employés de l'organisation, la constitution d'un plan de carrière personnalisé pour chacun des employés (et la responsabilisation de l'employé à son endroit), le développement de programmes de mentorat, l'élaboration de projets de fin de carrière pour les employés vieillissants, la mise sur pied d'un service 
de replacement des employés licenciés, la mise sur pied d'un service de réorientation de carrière et de développement professionnel, la valorisation des cheminements horizontaux de carrières et les changements volontaires d'unité de travail, la garantie de sécurité d'emploi à certains employés, le partage du temps de travail et enfin, l'entretien des contacts avec les anciens employés afin de leur faire parvenir de nouvelles possibilités de rejoindre les rangs de l'organisation. Toutes ces pratiques ne porteront vraisemblablement pas beaucoup de fruits si les gestionnaires licencient à la première occasion les employés qu'ils cherchent par ailleurs à fidéliser. Woodruffe (1999) mentionne à ce sujet qu'avant de procéder à quelque licenciement que ce soit, les gestionnaires devraient avoir fait la preuve à leurs employés que les actionnaires subissent depuis déjà longtemps les contrecoups d'un ralentissement économique majeur et que c'est en tout dernier recours qu'ils se voient obligés de couper des emplois.

La onzième famille de solutions est un amalgame de considérations liées à la communication organisationnelle, à la culture organisationnelle et au leadership des gestionnaires. Elle comporte encore une fois de nombreuses idées que nous diviserons, selon qu'elles sont plutôt classiques ou novatrices. Parmi les idées classiques fréquemment citées par les auteurs, notons la valorisation d'un leadership transformationnel inspiré de la théorie ' $\mathrm{Y}$ ' de McGregor, l'organisation régulière d'activités de socialisation et d'échanges entre les salariés et la direction, une charge de travail raisonnable pour les gestionnaires afin que ceux-ci puissent apporter leur support aux employés en difficulté, la valorisation d'une culture organisationnelle reconnaissant les mérites d'une gestion participative et honnête, l'élimination du port obligatoire de l'uniforme, la communication régulière des valeurs de l'organisation par le biais de journaux d'entreprise et de manuels des employés, la valorisation des rapports d'entraide entre collègues (notamment par le biais d'une politique qui leur laisse le soin de s'arranger à l'amiable en cas de pépin qui les empêcherait de bien faire leur boulot), une identification claire des rôles et responsabilités de chacun, le courage de prendre les décisions disciplinaires qui s'imposent et enfin, la mise sur place d'un système de boîtes de suggestions.
Parmi les solutions originales, notons l'organisation de journées de travail 'hyper chics' où tous les employés sont invités à se présenter au travail en tenue de gala, la création d'une culture organisationnelle forte où tous se sentent membres d'une même famille ou d'une même équipe sportive, la diffusion d'une politique qui interdit à quiconque de médire de qui que ce soit dans l'organisation, l'adoption d'une politique visant à augmenter les coûts d'une absence ou d'un retard au travail, l'adoption d'une politique visant à promouvoir le travail en équipe et 'condamner' les succès individuels et enfin, l'adoption d'une politique visant à interdire le présentéisme au sein de l'organisation.

Alors que pour Woodruffe (1999) tout cela revient à créer des cultures de l'engagement (cultures of commitment), pour Chambers (2001) tout cela revient à créer une véritable culture de la rétention du personnel. Plus spécifiquement, la culture de la rétention du personnel est composée selon lui d'une culture de la préparation des employés par le biais d'une formation continue, d'une culture de l'inclusion leur permettant de s'exprimer et de prendre part aux divers processus décisionnels, d'une culture du changement faisant prendre conscience à tous du besoin de se développer sans cesse et de s'adapter aux modifications nombreuses et rapides des environnements de l'organisation et enfin, d'une culture de la célébration visant à reconnaître le rendement aussi bien individuel que collectif et à s'assurer, par le biais de renforcements positifs, de la participation continuelle des employés aux processus en cours.

\section{Tout cela revient à créer des cultures de l'engagement.}

La douzième famille de solutions regroupe diverses idées liées à la détermination des conditions de travail. Dans cette famille, les divers auteurs recensés insistent pour que les gestionnaires s'assurent de répondre aux divers besoins des employés, sans pour autant privilégier un groupe au détriment d'un autre. Qu'il y ait ou non présence de l'acteur syndical, les gestionnaires devraient toujours permettre aux employés de négocier les conditions auxquelles ils acceptent de contribuer au succès de l'organisation. Enfin, afin de prévenir toute escalade des conflits, les gestionnaires auraient tout 
intérêt à créer une instance de résolution et de prévention des conflits au sein de l'organisation.

Il reste encore, dans le panier des solutions possibles pour prévenir tout roulement volontaire excessif de la main-d'œuvre, une piste centrale qui ne cadre pas dans les diverses pratiques de gestion présentées précédemment. Il s'agit de créer un Service d'attraction et de rétention de la main-d'œuvre. Relevant du PDG, le chef de ce service pourrait avoir la tâche de piloter l'ensemble de cette problématique et de tenir informé les autorités de l'évolution de la situation en temps réel. Ayant dans son coffre d'outils des 'stay interviews' (en plus des entrevues de départ et des enquêtes de satisfaction et de mobilisation des ressources humaines), son travail consisterait à créer pour l'entreprise une image de marque extrêmement attrayante aussi bien à l'interne qu'à l'externe de l'organisation. Pouvant se traduire par la fierté de travailler pour l'organisation ou d'en être client, cette image de marque serait sans doute l'aboutissement le plus complet de la résolution de cette problématique.

\section{Conclusion}

S'il y a un message important à retenir c'est que pour conserver ses employés et attirer d'excellentes candidatures en quantité importante l'organisation doit faire la démonstration qu'elle prend à cœur le bien-être de sa main-d'œuvre. Comme on aura pu le constater ce ne sont pas les solutions qui manquent aux problèmes d'attraction et de rétention. L'imagination de l'Homme est sans limite! De cette revue de la littérature il se dégage un constat essentiel : toutes ces solutions ne se valent pas complètement et donc, l'important n'est pas d'implanter toutes ces solutions, ou encore un ensemble particulier de ces dernières. L'important est de mettre en place les solutions que l'organisation peut se payer, qui correspondent notamment à sa culture organisationnelle, qui répondront aux besoins et aux attentes de la majorité de ses employés ou du moins, aux besoins et aux attentes des employés qu'elle ne veut pas perdre ou qu'elle ne peut pas se payer le luxe de perdre! Théoriquement parlant, il est aussi parfaitement concevable que chaque employé se voit attribuer, de manière préventive, un 'panier de moyens d'attraction et de rétention' au sein duquel il pourra faire un choix qui répondra à ses propres besoins. Sur le même principe que celui de l'assurance, tout dépendrait des coûts totaux des 'services choisis' et bien sûr, du montant que l'organisation est prête à consacrer à chacun de ces employés. Une simple étude des coûts actuels du roulement des employés pourra nous donner une très bonne idée des ressources financières dont pourrait disposer l'organisation pour mettre sur pied de tels paniers de solutions à la carte.

\section{L'organisation doit faire la démonstration qu'elle prend à cœur le bien-être de sa main- d'œuvre.}

\section{Notes et références}

1 Au sujet des organisations évoluant dans les secteurs 'les plus à risque' le lecteur est invité à examiner D'Ambroise, G. (2001). Rapport de recension d'écrits sur la problématique de l'attraction et de la rétention de la main-d'œuvre hautement qualifiée, Québec, Université Laval: Faculté des sciences de l'administration.). En ce qui a trait aux employés les plus susceptibles d'être touchés le lecteur est invité à examiner les contributions de Huselid, M. A., Becker, B.E. et Beatty, R.W. (2005). The Workforce Scorecard-Managing Human Capital to Execute Strategy. Boston, HBS et de Cappelli, P. A., 2002. Hiring and Keeping the Best People. Boston, HBS.

2 Pour une discussion intéressante de la façon dont les entreprises de restauration rapide profitent d'un taux de roulement élevé de leurs employés le lecteur est prié de consulter Nkouatchet, R.N., 2005, 'La précarité de l'emploi au service de la prospérité du fast-food', $\underline{\text { So- }}$ ciologie du travail, vol.47, pp.470-484.

3 En termes économiques ces idées font référence à ce que l'on appelle les marchés internes du travail. Gazier (2004) en présente d'anciennes mais superbes illustrations lorsqu'il aborde l'opposition Le CreusotlBillancourt entre 1900 et 1914 ((Gazier, B. (2004). Les salariés et les stratégies syndicales. Les stratégies syndicales des ressources humaines. É. L. Découverte. Paris: 79-97).

La bibliographie complète de cet article est disponible auprès de l'auteur (FrancoisBernard.Malo@rlt.ulaval.ca) 


\title{
Publicité
}

\author{
« 4 carrés »
}

